\title{
Systematic Analysis of MYB Family Genes in Potato and Their Multiple Roles in Development and Stress Responses
}

\author{
Xiaoxu Li ${ }^{1,2,+} \mathbb{1}$, Cun Guo ${ }^{1,3,+}$, Salman Ahmad ${ }^{1}$, Qi Wang ${ }^{1,3}$, Jing Yu ${ }^{1}$, Cheng Liu ${ }^{1}$ and \\ Yongfeng Guo 1,*(D) \\ 1 Key Laboratory for Tobacco Gene Resources, Tobacco Research Institute, Chinese Academy of Agricultural \\ Sciences, Qingdao 266101, China \\ 2 Technology Center, China Tobacco Hunan Industrial Co., Ltd., Changsha 410007, China \\ 3 Graduate School of Chinese Academy of Agricultural Sciences, Beijing 100081, China \\ * Correspondence: guoyongfeng@caas.cn; Tel.: +86-532-6671-5256 \\ + These authors contributed equally to this work.
}

Received: 21 June 2019; Accepted: 26 July 2019; Published: 30 July 2019

check for updates

\begin{abstract}
The MYB proteins represent a large family of transcription factors and play important roles in development, senescence, and stress responses in plants. In the current study, 233 MYB transcription factor-encoding genes were identified and analyzed in the potato genome, including 119 R1-MYB, 112 R2R3-MYB, and two R1R2R3-MYB members. R2R3-MYB is the most abundant MYB subclass and potato R2R3-MYB members together with their Arabidopsis homologs were divided into 35 well-supported subgroups as the result of phylogenetic analyses. Analyses on gene structure and protein motif revealed that members from the same subgroup shared similar exon/intron and motif organization, further supporting the results of phylogenetic analyses. Evolution of the potato MYB family was studied via syntenic analysis. Forty-one pairs of $S t M Y B$ genes were predicted to have arisen from tandem or segmental duplication events, which played important roles in the expansion of the $S t M Y B$ family. Expression profiling revealed that the $S t M Y B$ genes were expressed in various tissues and several $S T M Y B$ genes were identified to be induced by different stress conditions. Notably, StMYB030 was found to act as the homolog of AtMYB44 and was significantly up-regulated by salt and drought stress treatments. Furthermore, overexpression of StMYB030 in Arabidopsis enhanced salt stress tolerance of transgenic plants. The results from this study provided information for further functional analysis and for crop improvements through genetic manipulation of these StMYB genes.
\end{abstract}

Keywords: potato; MYB; biotic stress; abiotic stress; leaf senescence

\section{Introduction}

Transcription factors act as key regulatory components by switching the expression of genes involved in various biological processes [1]. The MYB family has been reported to be one of the largest transcription factor families in plants, regulating various developmental processes and stress responses [2,3]. MYB transcription factors harbor a conserved MYB domain at the $\mathrm{N}$ terminus, generally composed of one to four imperfect repeats, each possessing approximately 52 amino acids and forming three $\alpha$-helices structures. The second and third helices are often organized into a helix-turn-helix structure with three regularly spaced tryptophan residues, which play an important role in DNA binding [3,4]. Based on the number of adjacent repeats within the MYB domain, MYB proteins are classified into four groups, namely 1R-MYB, R2R3-MYB, R1R2R3-MYB, and 4R-MYB members containing one, two, three, and four MYB repeats, respectively. R2R3-MYB members are the most common subgroup of MYB members found in plants while in animals R1R2R3-MYB proteins are the 
predominant group [2,3]. It has been suggested that R2R3-MYB proteins have probably evolved from R1R2R3-MYB progenitors due to the loss of the R1 repeat [5].

In plants, the R2R3-MYB subfamily consists of majority of the MYB proteins and members in this subfamily play central roles in controlling plant-specific processes including plant development, secondary metabolism, and biotic/abiotic stress responses [3,6,7]. In Arabidopsis, the R2R3-MYB proteins have been classified into 25 subgroups and a number of $R 2 R 3-M Y B$ genes have been characterized to be involved in various developmental processes [3]. In subgroup 19, AtMYB21 and AtMYB24 control anther development, while AtMYB33 and AtMYB65 in subgroup18 facilitate both anther and pollen development [8-11]. AtMYB37/RAX1, AtMYB38/RAX2, and AtMYB84/RAX3 in subgroup 14 function in axillary meristem development as partially redundant regulators whereas AtMYB68 from the same subgroup specifically regulates root growth, influencing the whole plant development under harsh conditions [12]. In subgroup 15, AtMYB0 and AtMYB23 contribute to the origination of trichome in shoots while AtMYB23 acts in root hair patterning $[13,14]$. Being the last developmental process, leaf senescence could be triggered by age, phytohormones, and biotic/abiotic stresses while a large number of transcription factors, including members from the NAC, WRKY, and MYB family members, were reported to participate in leaf senescence [15]. In a previous study, AtMYB2 from subgroup 20 was found to be up-regulated during late stages of plant development and contributed to the regulation of whole plant senescence [16].

In addition, several R2R3-MYB members have been reported to be involved in regulation of metabolism in Arabidopsis [7]. In subgroup 7, AtMYB11/PFG2, AtMYB12/PFG1, and AtMYB111/PFG3 participate in biosynthesis of flavonols in all tissues [17]. In subgroup 6, AtMYB75/PAP1, AtMYB90/PAP2, and AtMYB113 regulate the biosynthesis of anthocyanin in vegetative tissues $[18,19]$. In subgroup 21, AtMYB52, AtMYB54, and AtMYB69 were suggested to be involved in biosynthesis of lignin, xylan, and cellulose [20,21]. Furthermore, in subgroup 12, AtMYB34/ATR1, AtMYB51/HIG1, and AtMYB122 control camalexin and indolic glucosinolates production in roots and late phase rosette leaves [22,23].

Furthermore, a large number of R2R3-MYB proteins were found to confer abiotic and/or biotic stress responses in Arabidopsis [6]. In subgroup 1, AtMYB60 and AtMYB96 control stomatal movement, drought stress, and disease resistance via the ABA signaling cascade while AtMYB30 confers a hypersensitive cell death response to disease attacks [24-27]. Similarly, AtMYB15 in subgroup 2, AtMYB33 in subgroup18, and AtMYB44 in subgroup 22 function in ABA-mediated responses to abiotic stresses and environmental signals [28-31]. In subgroup 20, AtMYB2 regulates plant response to dehydration and salinity stress after treating with exogenous ABA, AtMYB62 acts in phosphate starvation, while AtMYB108 was reported to be involved in responses to various biotic and abiotic stresses [32-35]. In defense response, AtMYB102 from subgroup 11 regulates resistance against herbivory and takes part in the response of Arabidopsis to aphid infestation, while AtMYB72 is a key regulator in activating induced systematic resistance upon colonization of roots by beneficial microorganisms [36-38].

Potato is an important food commodity worldwide, and the yield of potato is under constant threats induced by various stresses. The roles of MYB members in development and responses to biotic and abiotic stresses signify them to be potentially exploited in crop improvement. In addition to Arabidopsis, MYB family genes have been identified in a number of plant species, including tomato, grapes, rice, maize, chinese pear, sesame, and Tamarix hispida [39-45]. However, limited information is available about $M Y B$ family genes in potato. In the current study, a comprehensive analysis was carried out and the results revealed that the potato MYB family members may play multiple roles in potato development and in responses to various stresses. 


\section{Materials and Methods}

\subsection{Identification and Phylogenetic Analysis of Potato MYB Proteins}

The potato genome data (Release 3.4) and the Arabidopsis genome data were retrieved from the Sol Genomics Network (SGN, http://solgenomics.net/, Boyce Thompson Institute for Plant Research, NY, USA) and TAIR (Phoenix Bioinformatics Corporation, Fremont, CA, USA), respectively. The previously reported Arabidopsis MYB full-length protein sequences were used as queries to carry out BLASTP searches against the potato annotation database under the E-value cutoff of 0.1. The resulted sequences were then subjected to Pfam (https://pfam.xfam.org/, European Molecular Biology Laboratory, Cambridgeshire, UK) and SMART (http://smart.embl.de/, biobyte solutions GmbH, Heidelberg, Germany) analyses to detect the presence and number of the MYB domain. The resulted genes were named based on their physical locations across the 12 potato chromosomes. The ProtParam online toolkits (http://au.expasy.org/tools/protparam.html, Bioinformatics Resource Portal, Lausanne, Switzerland) were used to predict molecular weight and isoelectric point of candidate proteins.

The full-length protein sequences and MYB domain sequences of newly identified potato R2R3-MYB members together with the previously reported Arabidopsis R2R3-MYB members were subjected to perform multiple sequence alignments using MAFFT with the default settings. The alignment result of the R2R3-MYB domain was visualized by Texshade. A neighbor-joining (NJ) tree was constructed based on the prior full-length sequences alignment result using the MEGA package 6.06 with the following parameters: Poisson correction, 1000 replicate bootstrap values, and pairwise deletion. The tree file was visualized with FigTree 1.4.0 (University of Maryland, College Park, MD, USA).

\subsection{Motif and Gene Structure Analysis}

MEME (multiple expectation maximization for motif elicitation, http://meme-suite.org/, National Institutes of Health, Bethesda, MD, USA) was adopted for the identification of conserved motifs with the following parameters: Distribution of motif occurrences, zero or one per sequence; maximum number of motifs, 20; and the optimum width of each motif, between 6 and 100 residues [46].

The gene structure of Arabidopsis and potato R2R3-MYB genes were plotted by the gene structure display server (GSDS: http://gsds.cbi.pku.edu.cn, Beijing, China) by comparing the coding sequence (CDS) and genomic sequence obtained from the TAIR and SGN database, respectively [47].

\subsection{Chromosomal Localization and Duplication Event Analysis}

The chromosomal locations of the potato MYB genes were obtained from the SGN database and illustrated by Perl. The tandem gene duplications were identified as previously described [48]. The MCScanX program was used to identify segmental duplications based on the previous reports and the results were visualized by Circos [43]. To explore the synteny relationship of the orthologous genes obtained from potato and other tested plant species, the syntenic analysis maps were generated by the Systeny Plotter of Tbtools (South China Agricultural University, Guangzhou, China), as reported previously [49]. The non-synonymous ( $\mathrm{ka}$ ) and synonymous (ks) substitutions of each duplicated gene pair were calculated by DnaSP v5 (Universitat de Barcelona, Barcelona, Spain) [50].

\subsection{Expression Profiling of Potato MYB Genes}

The RNA-seq data of StMYB genes were retrieved from the International Potato Genome Sequencing Consortium (PGSC, http://solanaceae.plantbiology.msu.edu/, Michigan State University, MI, USA) that were generated in a previous study [51]. The expression data of root, stem, shoot apex, leaf, stolon, flower, and tuber were selected. The relative expression ratios of biotic and abiotic stress treatments were calculated relative to their controls, respectively. The StMYB gene expression data were normalized and visualized by $R$. 


\subsection{Growth and Stress Treatments of Potato Plants}

Shoot cultures of potato Cultivar GN2 were used in this study. Shoots were inoculated and cultured on MS solid-media via nodal cutting. Potato seedlings were maintained in a growth chamber at $22{ }^{\circ} \mathrm{C}$ under $16 \mathrm{~h}$ light with $8 \mathrm{~h}$ dark for two weeks. For salt stress treatments, the seedlings were treated with $150 \mathrm{mM} \mathrm{NaCl}$, as previously reported [48]. Further, these cultures were transferred into the soil in the same growth chamber for two months to obtain tuber. Different tissues, including shoot, shoot tip, root, young leaves, senescent leaves, and tuber were used in exploring the tissue-specific expression patterns. All of these tested samples were frozen by the liquid nitrogen and transferred to $-80{ }^{\circ} \mathrm{C}$ immediately.

\subsection{RNA Extraction and $q R T-P C R$ Analysis}

Total RNA of tested sample was extracted by Trizol, in combination with RNase-free DNase treatment. Total RNA was used for the synthesis of first strand of cDNA by using the PrimeScript ${ }^{\mathrm{TM}} \mathrm{RT}$ reagent Kit (TaKaRa, Shiga, Japan) according to the manufacturer's instructions. The RT-qPCR was performed on an ABI 7500 real-time PCR instrument (Applied Biosystems, Waltham, MA, USA). The potato Elongation Factor 1- $\alpha$ (EF1 $\alpha)$ gene was adopted as the internal control [52]. RT-qPCR of each gene was performed with three biological replications and the resulting data were collected and analyzed by the $2^{-\Delta \Delta C T}$ method. The primer sequences used in the current study were listed in Supplementary Table S1.

\subsection{Subcellular Localization}

The CDS of StMYB030 without stop codon and the GFP fragment were amplified and inserted into the $\mathrm{PCHF} 3$ vector by Infusion (Invitrogen) separately, generating the StMYB030-GFP fusion fragment driven by the CaMV-35S promoter. The recombinant vector was introduced into Agrobacterium strain GV3101 for transient expression in the leaf of Nicotiana benthamiana. After incubation of 2-4 days, these leaves were subjected to 4,6-diamidino-2-phenylindole (DAPI) staining to confirm the position of the nucleus. As previous studies, the confocal microscope (TCS-SP8 Leica, Wetzlar, Germany) was used to capture the fluorescence signals $[53,54]$.

\subsection{Overexpression Analysis}

The coding sequence of StMYB030 gene was amplified and ligated into the pCHF3 by Infusion (Invitrogen, Carlsbad, CA, USA), which was under the control of the CaMV-35S promoter. This recombinant construct was introduced into Agrobacterium strain GV3101 and transformed into Arabidopsis Col-0 plants by the floral dip method [55]. The positive transgenic lines were screened out on half MS solid plates with $50 \mathrm{mg} / \mathrm{L}$ kanamycin. The T3 generation lines were generated and selected for further analysis. The Arabidopsis wildtype Col-0 and two StMYB030-overexpression transgenic lines were sown on half MS solid plates and grown vertically for seven days. Then, these seedlings were transferred to new half MS solid plates supplemented with 0 or $100 \mathrm{mM} \mathrm{NaCl}$. All plants were grown under continuous light at $22{ }^{\circ} \mathrm{C}$. The primary root length was measured at 14 days after treatment. Three independent biological replicates each containing five plants were conducted for each treatment. The $t$-tests were performed with GraphPad Prism 5 (GraphPad Software Inc., San Diego, CA, USA).

\section{Results}

\subsection{Identification of MYB Genes in Potato}

To identify MYB genes in potato, the BLASTP search was performed using the full-length sequences of the reported Arabidopsis MYB members as queries. Then, the resulted hits were subjected to Pfam and SMART analyses, surveying the presence and the repeat number of complete MYB motif in each candidate. As a result, a total of 233 MYB genes were identified, including 119 R1-MYB, 112 R2R3-MYB, 
and 2 R1R2R3 MYB members. No 4R-MYB member was detected in potato. We were able to map 230 of the newly identified $M Y B$ genes on the 12 chromosomes of potato and named them with the prefix 'St' implying Solanum tuberosum, followed by numbers in the order of physical position from top to bottom of each chromosome, starting from chromosome 1 . Three of the MYB genes were not mapped to any chromosome and were named StMYB231, StMYB232, and StMYB233, respectively.

The detailed information, including length of CDS, protein molecular weight (MW), and isoelectric point (pI) were analyzed. Among the 233 identified StMYB members, StMYB009 was found to be the smallest MYB protein, whereas the largest MYB protein was StMYB209 with more than 1000 aa. The molecular weight of the identified members ranged from 7.4 to $113.4 \mathrm{kDa}$, whereas the pI ranged from 4.23 to 10.27. The detailed information could be available in Supplementary Table S2.

\subsection{Multiple Sequence Alignment and Phylogenetic Analysis}

In the plant kingdom, the R2R3-MYB is the largest subgroup of MYB proteins that harbors a highly conserved DNA-binding domain composed of two adjacent MYB repeats [5]. To explore the characteristics of the potato R2R3-MYB members, the MYB domain of the potato R2R3-MYB members were extracted and subjected to multiple sequence alignment. These results revealed that the potato R2 and R3 MYB domains harbor the conserved amino acid residues, particularly the characteristic Trp. Three highly conserved Trp residues were observed in the R2 MYB domain. In the potato R3 MYB domain, only the second and third Trp were found to be conserved, whereas the first Trp was substituted by Phe or Ile (Figure 1A). In addition, the sequence features of the R2R3-MYB domain were highly conserved between the potato and Arabidopsis members (Figure 1B).

A
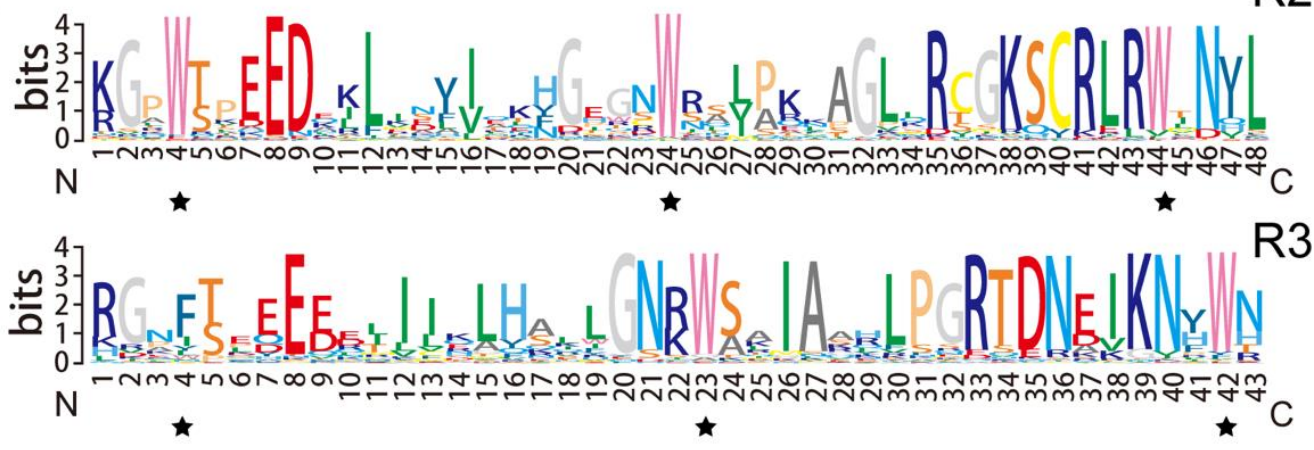

B
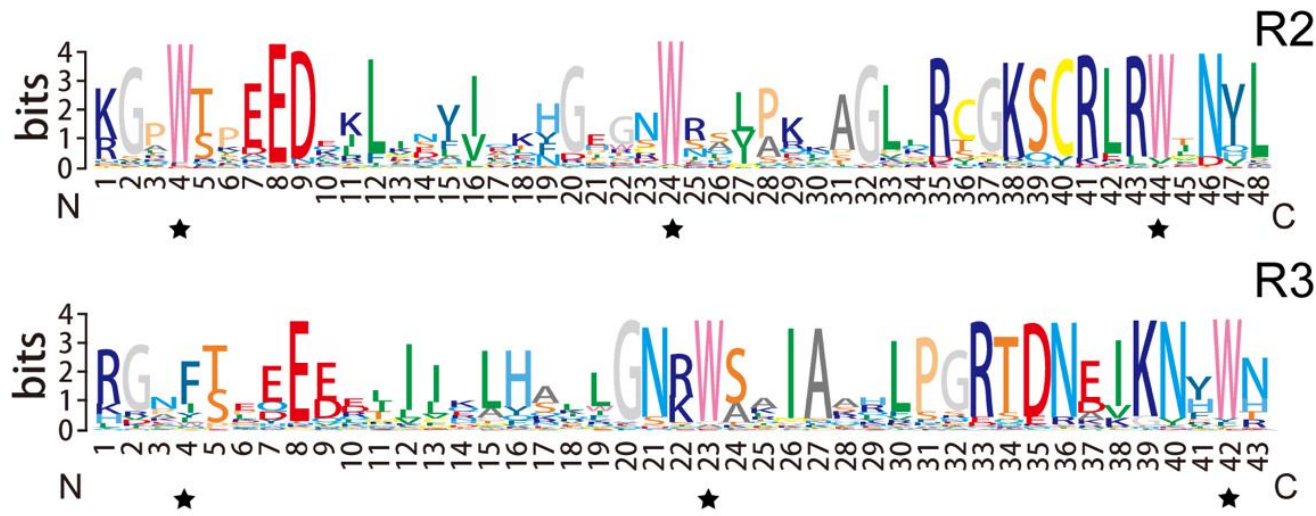

Figure 1. Sequence logos of the R2 and R3 MYB repeats of MYB members from potato (A) and Arabidopsis (B). The results were generated by multiple alignment analysis of Arabidopsis and potato R2R3-MYB members and visualized by Texshade. The asterisks indicate the typical conserved Trp residues in the MYB domain. 
R2R3-MYB members are the most common subgroup of MYB members found in plants [2,3]. The multiple sequence alignment of 112 potato R2R3-MYB members and their Arabidopsis homologs was performed and generated a neighbor-joining tree based on the alignment (Figure 2). The results showed that all the R2R3-MYB members from potato and Arabidopsis fell into 34 subgroups, of which S1 to S25 subgroups were highly consistent with the previous reports and were named based on the earlier systems [3]. The rest of the subgroups were named from S26 to S34, which contained some of the Arabidopsis R2R3-MYB members that were originally not included in the 25 subgroups. It is worth mentioning that most of the subgroups possessed members from both potato and Arabidopsis, indicating that the expansion of R2R3-MYB members may appear before the divergence of potato and Arabidopsis. Intriguingly, it was found that Subgroup 29 only harbored R2R3-MYB members from potato and Subgroup 12 only contained members from Arabidopsis.

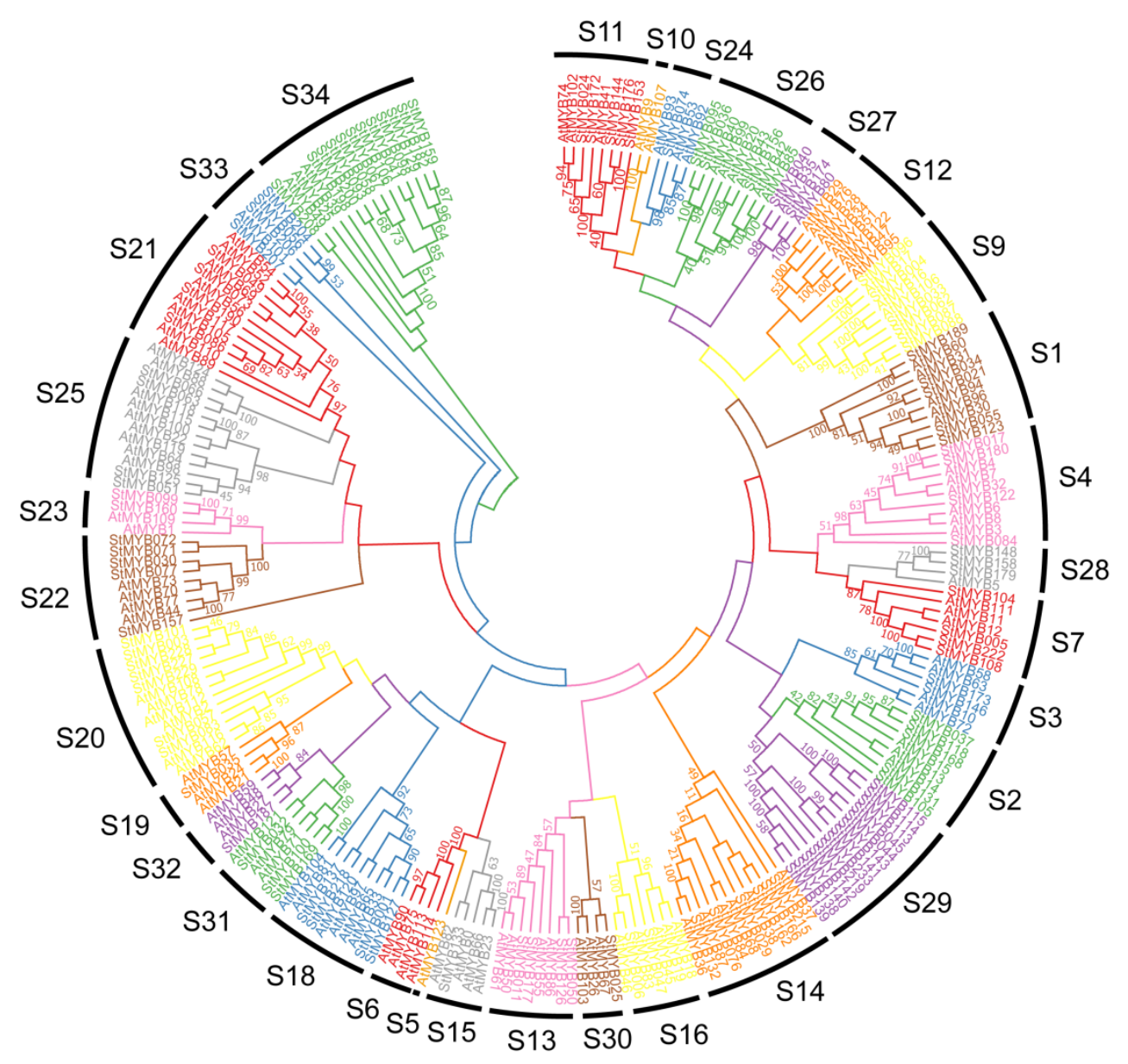

Figure 2. Phylogenetic analysis of potato R2R3-MYB family members. The phylogenetic tree was generated from the alignment of potato and Arabidopsis R2R3-MYB proteins with 1000 bootstrap replicates using the neighbor-joining (NJ) method. The potato R2R3-MYB members together with their Arabidopsis homologs were classified into 34 subgroups. 


\subsection{Gene Structure and Motif Composition}

The gene structure could provide evolutional information. To gain insights into the evolution of the R2R3-MYB family in potato, the number and arrangement of intron-exon were identified in 112 potato R2R3-MYB genes and their Arabidopsis homologs. The results showed that genes within the same group usually have the similar intro-exon organization, and that the StR2R3-MYB genes shared the similar gene structures with Arabidopsis members in the same group (Supplementary Figure S1). Among StR2R3-MYB genes, the number of introns varied from 0 to 10, with the majority genes having two introns (66\%) or one intron (21\%). Three introns were found in four StMYB genes whereas 6, 9, and 10 introns were found in StMYB208, StMYB063, and StMYB066, respectively. Furthermore, six $S t M Y B$ genes were found to be intron-less.

The conserved motifs of StMYB proteins were predicted by the MEME program and a total of 20 distinct motifs were identified. The motifs were named motif $1-20$, among which motif $3,4,1$, and 2 together formed the R2R3-MYB domain (Supplementary Figures S1 and S2). The potato R2R3-MYB members were featured with two conserved MYB domain repeats, motifs $3 / 4$ and motif $1 / 2$ were identified as the R2 MYB repeat and the R3 MYB repeat respectively, while motif 1 may be substituted by motif 8 or 17. Inconsistent with the results of phylogenetic analysis, StMYB members within the same group were found to share the similar motif organizations. Notably, motif 14 was only found in subgroup 20, motif 15 was conserved in subgroup 1 , and motif 20 was unique in subgroup 29 . These unique motifs may contribute to functional divergence.

\subsection{Syntenic Analysis}

To further explore evolution of the potato MYB family members, syntenic analysis was performed between potato and five other plant species, including tomato, grape, and Arabidopsis representing dicot species, while rice and maize represented monocot species (Figure 3A). The results revealed syntenic relationship between 189 of the StMYB genes with MYB genes in tomato, followed by 136 StMYB genes with grape, 88 StMYB genes with Arabidopsis, 29 and 14 StMYB genes with MYB genes from maize and rice, respectively. Notably, more than half of the predicted collinear pairs between potato and tomato, both belonging to the Solanaceae family, are localized at highly conserved syntenic regions comprising more than 100 syntenic genes, whereas all of the predicted collinear pairs between potato and maize were found in syntenic blocks harboring less than 10 pairs. A total of 11 StMYB genes were predicted to form collinear pairs with genes from all of the other five species, suggesting that these genes may have existed before the divergence of these plant species (Figure 3B). Interestingly, 69 collinear pairs identified between potato and tomato/grape/Arabidopsis were not found with rice/maize, implying those pairs may have appeared after the divergence of dicot and monocot species. Notably, 25 AtMYB genes were predicted to be paired with at least two potato genes, suggesting that these additional potato genes may have arisen from duplication events and may play important roles during evolution in potato. The detailed information of syntenic gene pairs is provided in Supplementary Table S3. 
A
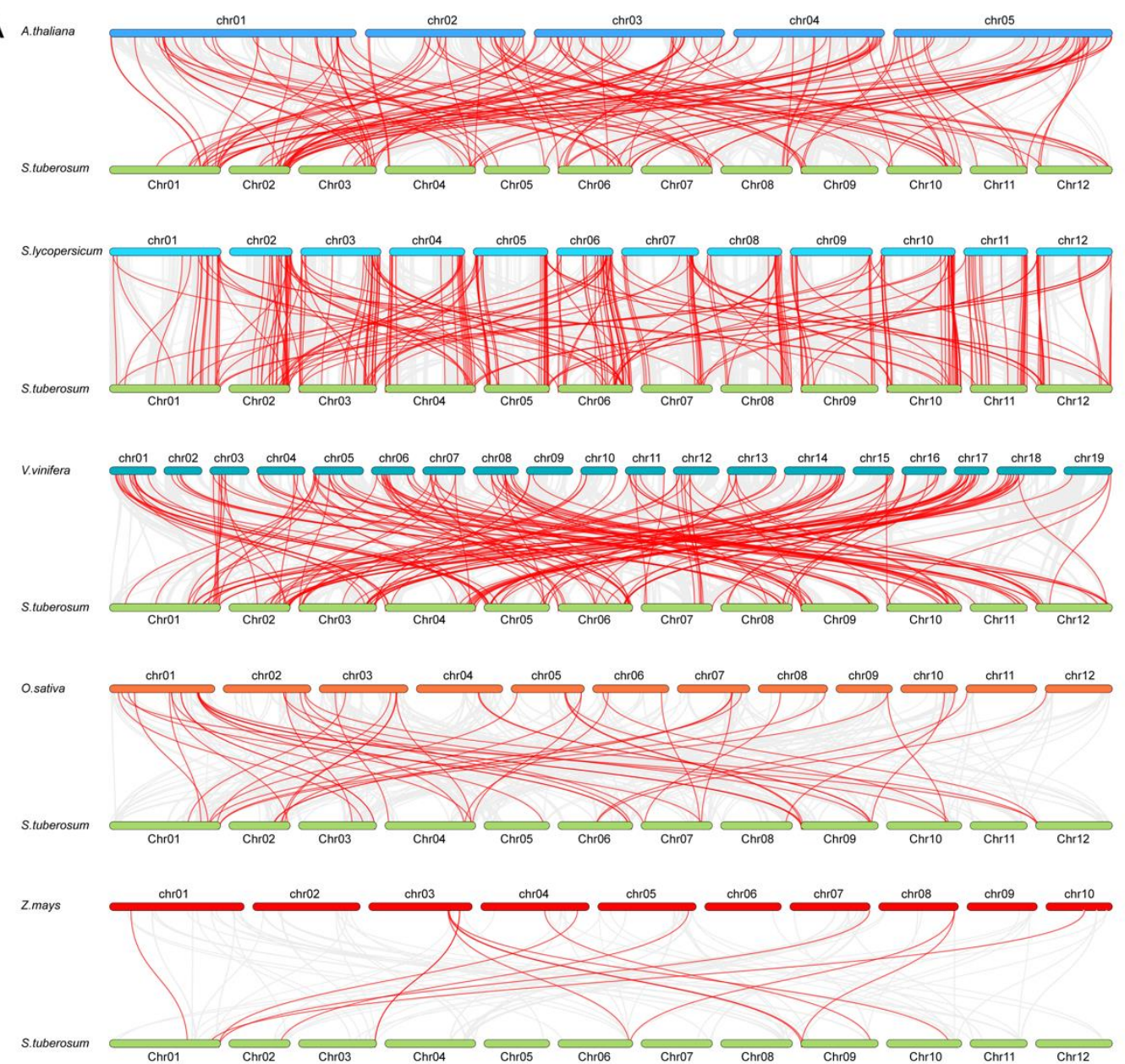

B

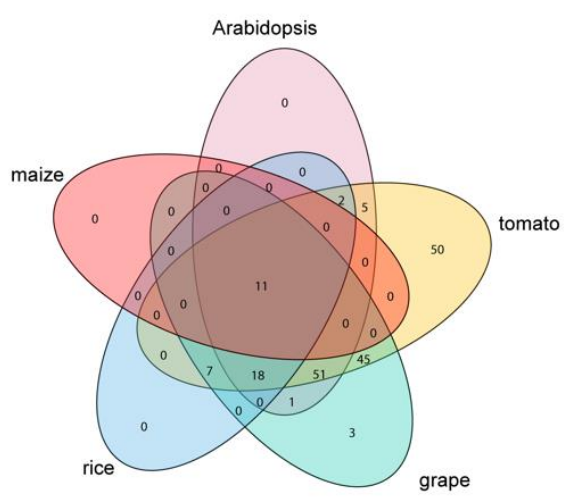

Figure 3. Synteny analysis of $M Y B$ genes between potato and five other representative species. (A) The gray line in the background represented the collinear blocks between potato and five other representative species, while the red line exhibited the syntenic MYB gene pairs. (B) The MYB genes formed the syntenic pairs between potato and all the other five selected species, which was visualized by the Venn plot.

\subsection{Chromosomal Distribution and Duplication Events}

The 230 StMYB genes mapped on potato chromosomes were unevenly distributed on the 12 chromosomes (Figure 4). The chromosome 5 harbored the most (26) and the chromosomes 08 and 09 harbored the least number (12) of potato $M Y B$ family genes. As defined in an earlier study [48], adjacent StMYB genes located within $200 \mathrm{~kb}$ were defined as forming a cluster, and StMYB genes sharing more than $70 \%$ identity in a cluster were considered as tandem duplication genes. A total of 
76 StMYB genes were thereby identified to form 29 clusters on different chromosomes. In addition, eight tandem duplication arrays consisting of $18 S t M Y B$ genes were identified.

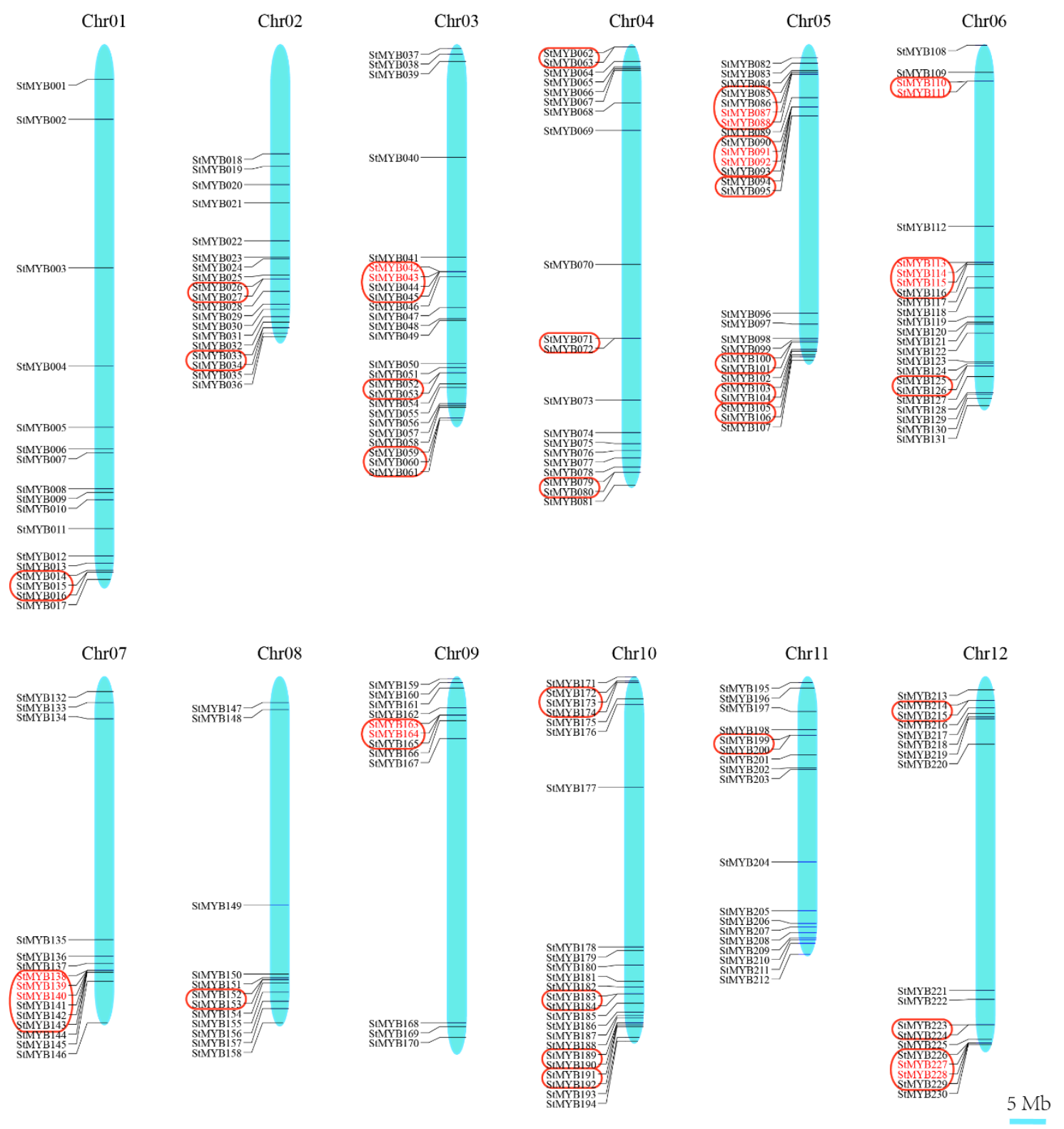

Figure 4. Distribution of StMYB genes on potato chromosomes. In potato, 230 StMYB genes were successfully mapped to 12 potato chromosomes. The red box indicated the gene cluster, while the tandem duplication pair was featured by red color.

Furthermore, segmental duplication or whole genome duplication analysis of the StMYB genes was performed using the MCScanX. Totally, 31 segmental duplication pairs with 57 StMYB genes were identified (Figure 5). These results suggested that about $32.2 \%$ of the $S t M Y B$ genes may be generated by duplication events, which played the major role in the expansion of the MYB gene family in potato. All of the tandem and segmental duplication genes were listed in Supplementary Table S4. 


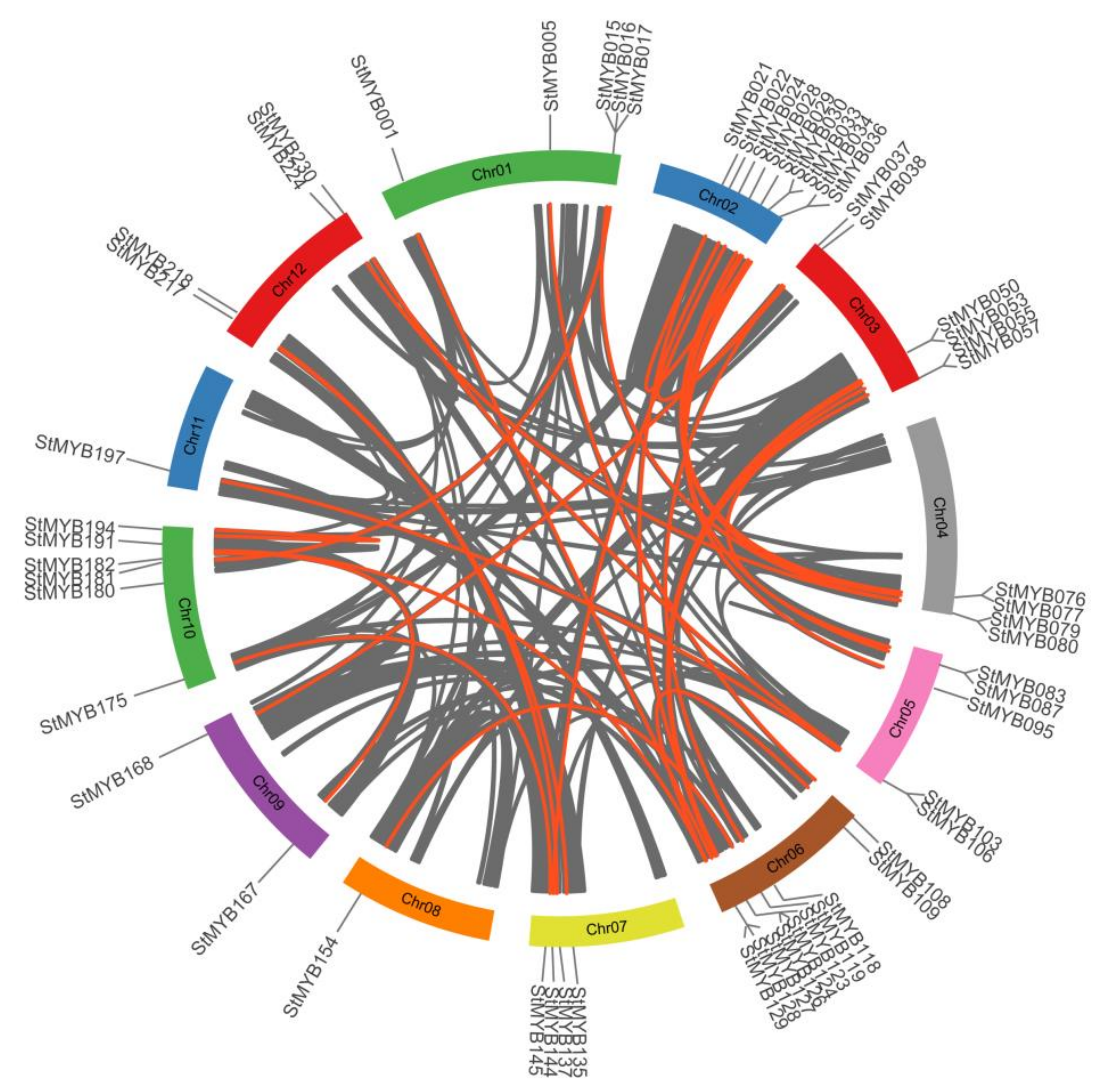

Figure 5. Segmental duplication events and inter-chromosomal relationships between StMYB genes. The 31 putative segmental duplication pairs of StMYB genes were investigated with MCScanX and linked by the colored lines, respectively. The gray lines indicate all putative segmental duplication pairs in the potato genome.

$\mathrm{The} \mathrm{Ka} / \mathrm{Ks}$ ratio is used to estimate the balance between neutral mutations, purifying selection, and beneficial mutations. The $\mathrm{Ka} / \mathrm{Ks}$ ratios of the tandem and segmental duplication gene pairs were calculated. As a result, all of the $\mathrm{Ka} / \mathrm{Ks}$ ratios were less than 1 , suggesting that the potato $M Y B$ duplicated members may have experienced purifying selective pressure during evolution.

\subsection{Promoter Analysis of StMYB Genes}

To study the expression regulation of the $S t M Y B$ genes, putative cis-elements on promoter regions were predicted using PlantCARE (Figure 6). Cis-elements related to developmental processes, such as meristem development (CAT-box), were detected in promoter regions of certain StMYB genes. The hormone-responsive elements were also identified in the promoters, including ABRE, ERE, TCA-element, CGTCA-motif, and AuxRR-core, which mediate the plant responses to ABA, ethylene, salicylic acid (SA), methyl jasmonate (MeJA), and auxin, respectively. Interestingly, the ABRE element was detected in promoter regions of most of the StMYB genes, implying those StMYB genes might participate in ABA-mediated stress responses. Furthermore, stress-responsive elements including WRKY binding site (W-box), MYB binding site (MBS), stress-responsive element (TC-rich repeats), heat stress-responsive element (HSE), low-temperature-responsive element (LTR), wound-responsive element (WUN-motif), and anaerobic induction element (ARE) were observed to be abundant in the promoters of a large number of $S t M Y B$ genes, suggested that these genes may participate in the responses to various stress conditions. 


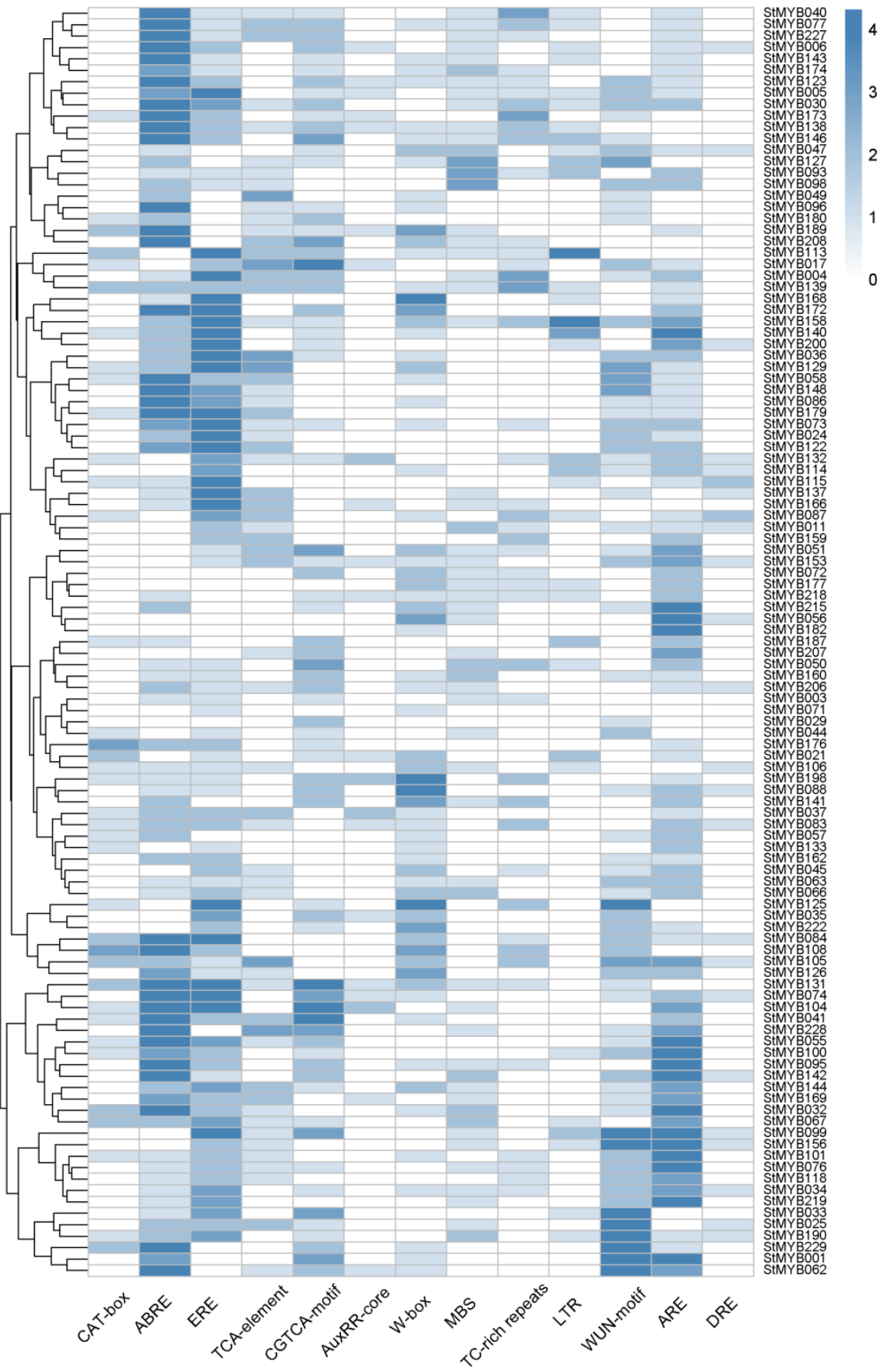

Figure 6. Regulatory elements in the promoter regions of StMYB genes.

\subsection{Expression of StMYB Genes in Different Tissue Types}

The gene expression patterns could provide information for potential gene function. The RNA-Seq data for $S t M Y B$ genes in eight representative tissues, including root, stem shoot apex, stolon, leaf, flower, young tuber, and mature tuber, were obtained from the PGSC and analyzed [51]. The expression data for 171 StMYB genes were detected in at least one of the eight tested tissues, with a large number of $S t M Y B$ genes being highly expressed in all eight tissues (Figure 7). While, a number of StMYB genes exhibited tissue-specific expression pattern either in only one or more tissues. For example, StMYB169 and StMYB224 were expressed exclusively in roots. The expression of StMYB006, StMYB049, StMYB126, and StMYB183 was detected in the stem only. Other tissue-specific expression patterns were also detected, including StMYB004, StMYB08.7 and StMYB214 in the shoot apex, StMYB035, 
StMYB039, and StMYB116 in flowers, as well as StMYB074 in mature tubers. In addition, StMYB036 and StMYB177 were highly expressed in stem/stolon, StMYB023 and StMYB096 were highly expressed in shoot apex/flower, while high-level expression of StMYB162, StMYB166, and StMYB181 was detected in root, young, and mature tuber tissues.

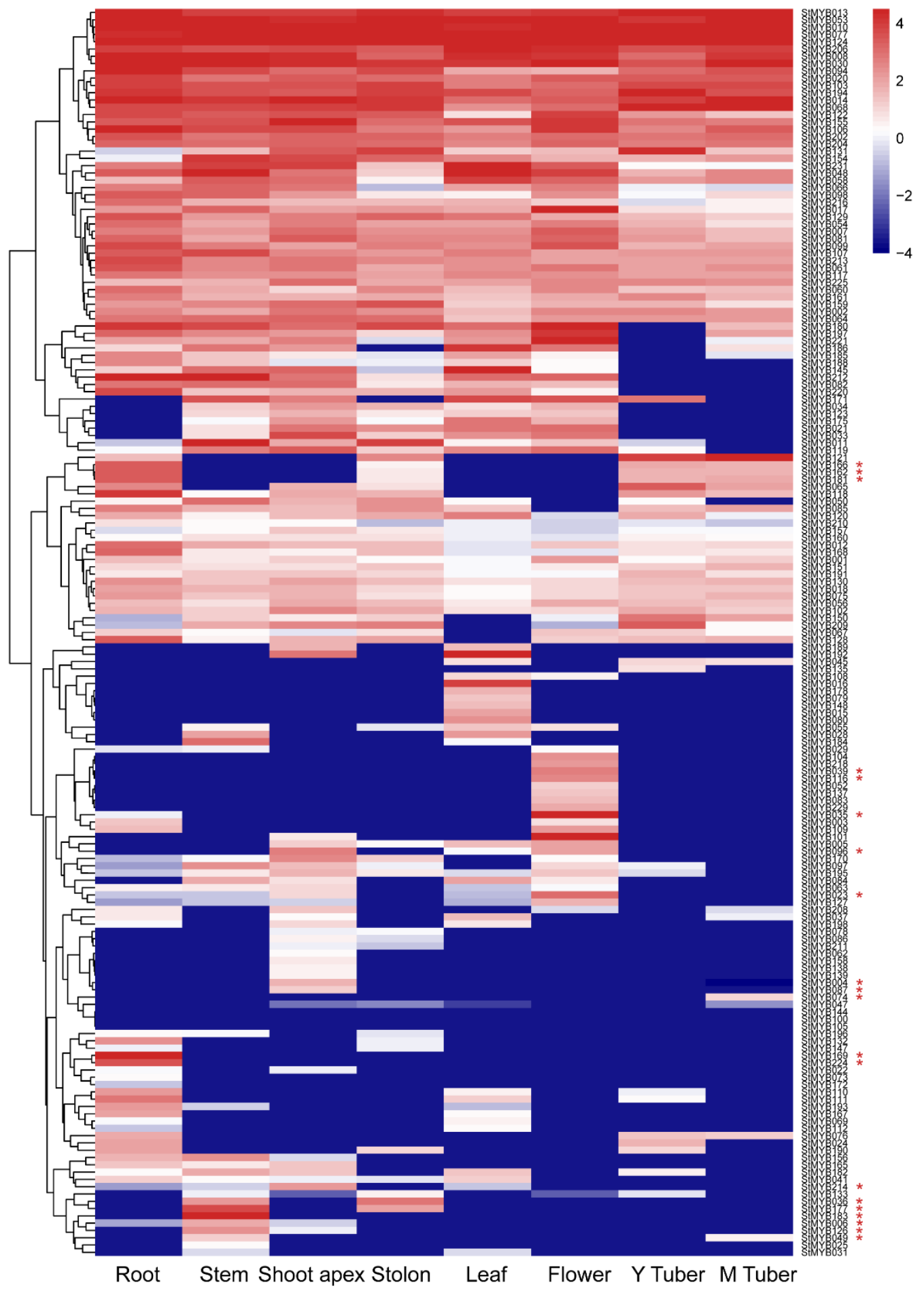

Figure 7. The expression patterns of the $S t M Y B$ genes in the tested tissues. The expression pattern data were retrieved from transcriptome data and visualized by R Programming Language. $Y$ Tuber, young tuber; M Tuber, mature tuber. 


\subsection{Expression Patterns of StMYB Genes in Response to Stress Treatments}

To investigate the stress-responsiveness StMYB genes, the RNA-Seq data for different abiotic stress treatments including salinity, drought, heat, and wounding were analyzed (Figure 8). As a result, a total of 80 StMYB genes were significantly induced under one or more of these treatments (Figure 8A), among which, StMYB080, StMYB110, and StMYB112 were highly expressed under all the four stress treatments, whereas, StMYB037, StMYB063, StMYB066, StMYB144, StMYB166, StMYB168, and StMYB212 were induced by all the stress conditions except wounding. Furthermore, StMYB048 and StMYB111 showed higher expression in responses to heat and wounding. Further, StMYB069, StMYB145, StMYB171, StMYB175, and StMYB186 were induced only after heat and wounding treatments while StMYB030, StMYB110, StMYB144, StMYB147, and StMYB150 were highly expressed in response to salt and drought stresses.

The expression changes of $S t M Y B$ genes in response to biotic stresses were also analyzed. RNA-seq data from Phytophthora infestans inoculation and application of two chemical elicitors, BABA and BTH were used for analysis (Figure $8 \mathrm{~B}$ ). The results showed that none of the StMYB gene was explicitly expressed after treatments of all three biotic stresses. A total of 20 StMYB genes were induced by one or more of the biotic stress conditions. StMYB022 was found to be up-regulated under both BABA treatment and P. infestans inoculation. StMYB225 was only induced by P. infestans inoculation. Expression of StMYB128 and StMYB155 was induced by BABA treatment only, whereas StMYB077, StMYB122, StMYB124, and StMYB210 were induced only by BTH treatment.
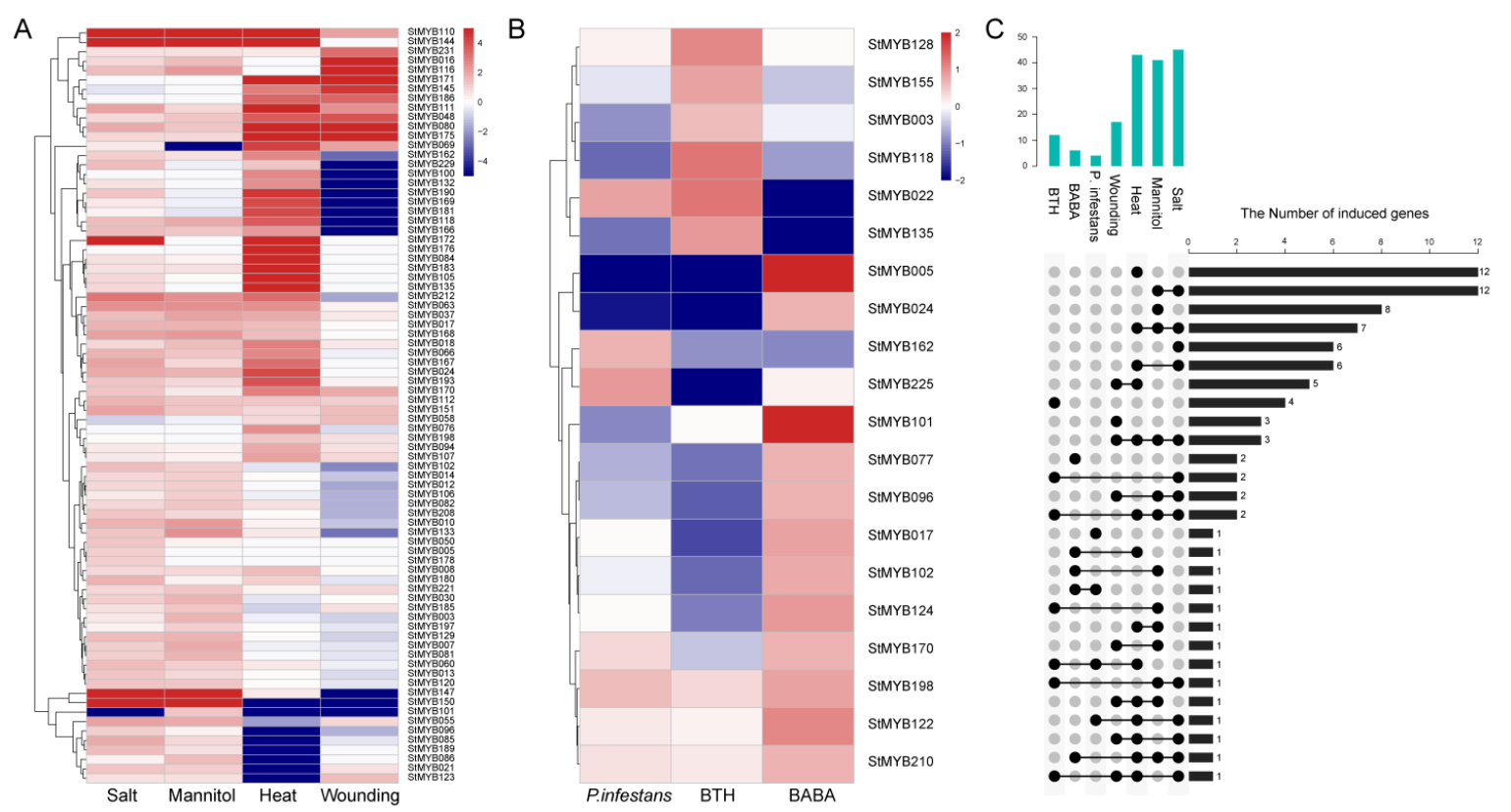

Figure 8. The expression patterns of $S t M Y B$ genes under abiotic and biotic stress treatments. (A) The relative expression ratios of abiotic stress treatments; (B) the relative expression ratios of biotic stress treatments; (C) the summarized information of the stress-induced StMYB genes. The relative expression ratios of abiotic and biotic stress treatments were calculated relative to the untreated control, respectively, and then the significantly induced gene was defined to possess a $\log 2$ relative expression ratio $\geq 1$ under one of the stress treatments. The red color, white color, and blue color represent the up-regulated, unaltered, and down-regulated expression, respectively.

Moreover, a number of $S t M Y B$ genes were induced under both abiotic and biotic stress conditions (Figure 8C). For example, StMYB118 was found to be induced by BABA and under all the tested abiotic stress treatments except wounding. StMYB017, StMYB024, and StMYB170 were up-regulated by BTH and in response to salt and heat treatments. Similarly, StMYB162 was highly induced by $P$. infestans inoculation and under heat and salt stress treatments. The numbers of StMYB genes that 
were significantly induced by specific stress treatment are summarized in Supplementary Table S5, including 8 genes induced by drought, 12 induced by heat, 3 induced by wounding, 1 induced by $P$. infestans, 2 induced by BABA, and 3 induced by BTH treatment, respectively (Figure 8C and Supplementary Table S5).

\subsection{Validation of Expression Patterns by qRT-PCR}

To confirm the expression changes of the $S t M Y B$ genes from RNA-seq analysis, representative $S t M Y B$ genes were selected to perform qRT-PCR analysis (Figure 9). Overall, the qRT-PCR data of representative genes agreed with the results of the RNA-seq analysis. A minor difference exists potentially due to variation caused by samples collecting methods and difference in developmental status.

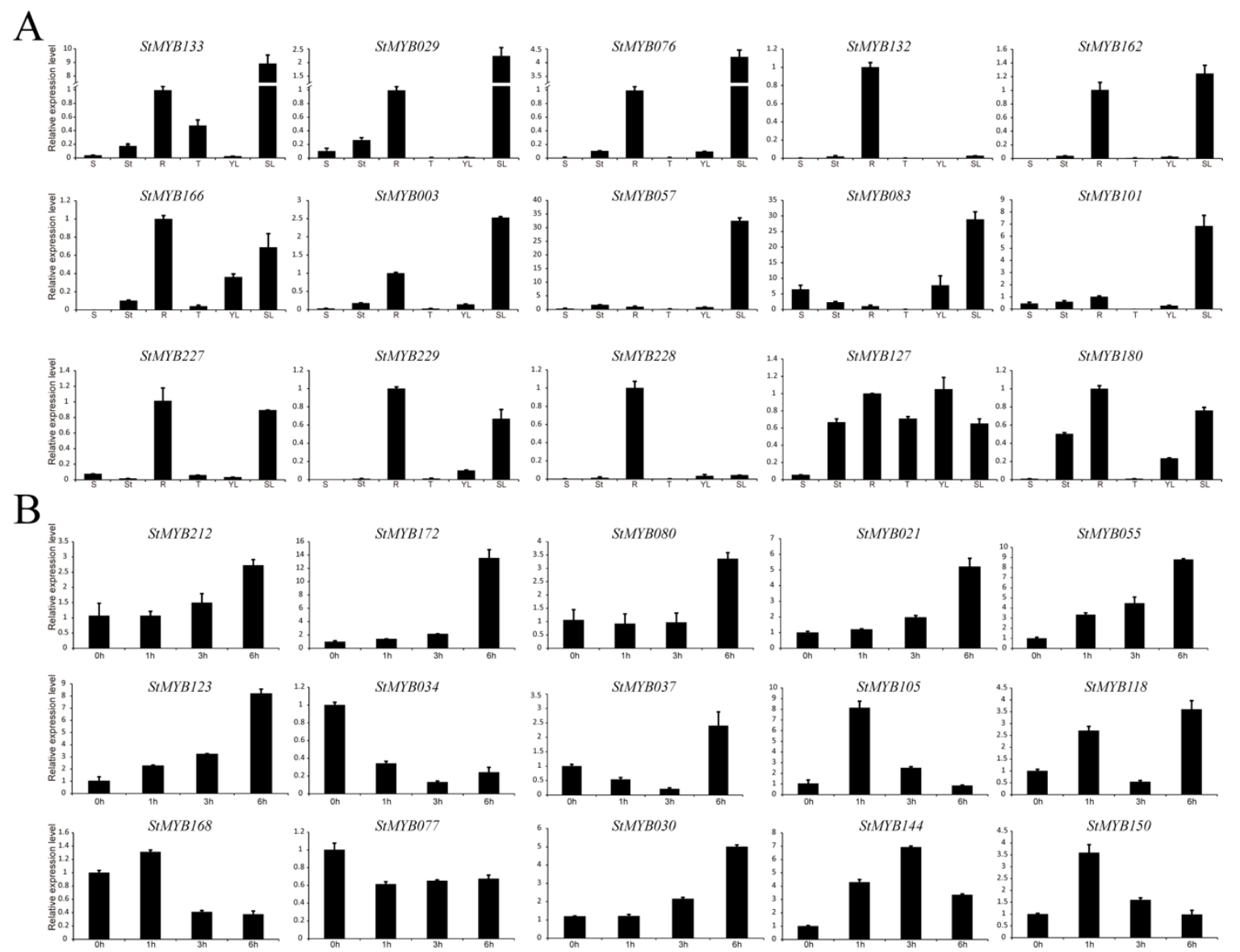

Figure 9. The expression patterns of selected StMYB genes detected by qRT-PCR. (A) To confirm the tissue specificity, the expression pattern of the selected $S t M Y B$ genes was calculated as folds relative to the expression level of the root. (B) The expression pattern of selected StMYB genes in response to salt stress treatments, which was calculated as folds relative to the untreated control.

As results, StMYB133 was found to express in the root, shoot tip, tuber, and senescent leaf specifically (Figure 9A). In subgroup 14, StMYB029, StMYB076, StMYB132, StMYB162, and StMYB166 were found to share similar expression pattern and highly expressed in the root, indicted these homologs may act redundantly. In subgroup 20, StMYB003, StMYB057, StMYB083, StMYB101, StMYB227, and StMYB229 were observed to highly expressed in senescent leaf. Notably, StMYB228 were also grouped with $A t M Y B 2$ in subgroup 20, which did not show abundant transcripts in senescent leaf.

Furthermore, inconsistent with the results from transcriptome data, several StMYB genes were significantly induced by salt treatments, such as StMYB212, StMYB172, and StMYB080 (Figure 9B). In 
subgroup 1, StMYB021, StMYB055, and StMYB123 significantly responded to salt treatments, whereas StMYB034 were repressed by salt. Furthermore, in subgroup 2, StMYB037, StMYB105, StMYB118, and $S t M Y B 168$ responded to the salt treatment in a different manner. Interestingly, in subgroup 22, StMYB077 was down-regulated by salt stress treatment, whereas StMYB030 was significantly induced by salt treatment.

\subsection{Subcellular Localization Analysis}

To further explore the potential function of the StMYB genes, the subcellular localization of the one of the stress-responsive genes, StMYB030, was analyzed (Figure 10). The coding sequence of StMYB030 without the stop codon was fused with the GFP reporter gene, which was driven by the CaMV35S promoter. The Agrobacterium cultures with the recombinant construct and the 35S::GFP control were used to inject the tobacco leaf epidermal cells. As revealed by confocal microscopy, the green fluorescence of the StMYB030-GFP fusion protein was specifically confined within the nucleus, which was further verified by DAPI staining, whereas the signal of GFP protein was found to distribute throughout the whole cell.
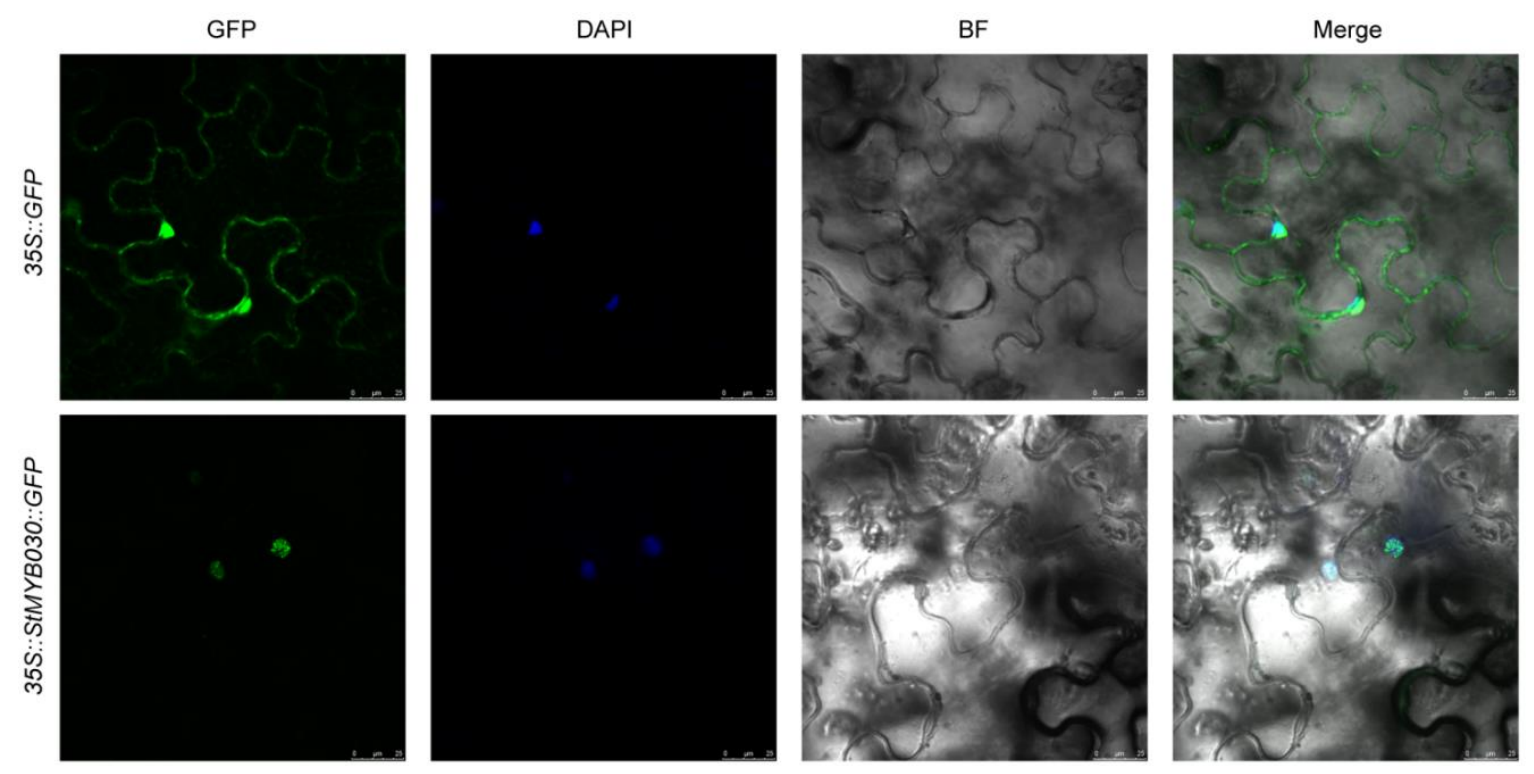

Figure 10. The subcellular localization of StMYB030 in tobacco epidermal cells. The StMYB030-GFP fusion construct and GFP gene driven by the CaMV-35S promoter were transiently expressed into tobacco, respectively. DAPI (dye 4,6-diamidino-2-phenylindole) staining indicted the nucleus.

\subsection{Overexpression of StMYB030 Gene Enhanced Salt Tolerance in Arabidopsis}

The function of StMYB030 gene was further examined via ectopic expression in Arabidopsis. The root elongation assay was performed to examine the salt tolerance of wildtype and StMYB030 overexpressing Arabidopsis (Figure 11 and Supplementary Figure S3). There was no significant difference in root length between wildtype and the StMYB030 overexpressing plants under normal conditions. However, compared to the wildtype, significantly longer roots of the transgenic plants were observed after 14 days growing on $100 \mathrm{mM} \mathrm{NaCl}$ plates. Then, two independent overexpression lines displayed the similar phenotype with longer roots under salt stress treatments. Taken together, these results indicated that the overexpression of StMYB030 gene improved the salt tolerance in transgenic Arabidopsis. 

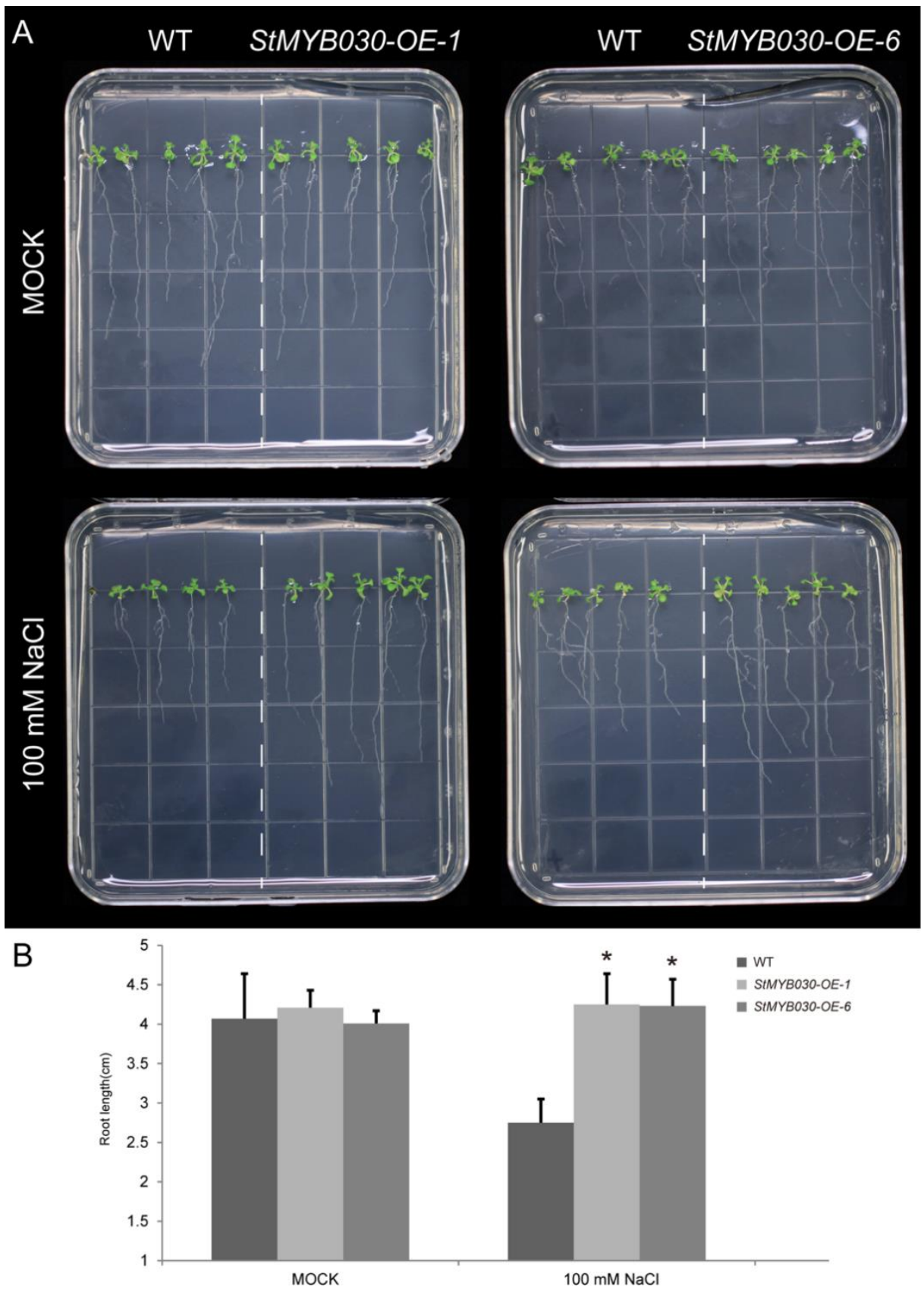

Figure 11. The Effects of salt stress treatment on root growth of StMYB030 gene overexpressing lines in transgenic Arabidopsis. (A) The primary root length of wildtype and StMYB030 gene overexpression lines under salt treatments in transgenic Arabidopsis. (B) The quantification of primary root length under normal condition and $100 \mathrm{mM} \mathrm{NaCl}$ treatments. The data were retrieved from three biological replicates. WT, wildtype. The data were means $\pm \mathrm{SD}$ of three biological repeats. ${ }^{*} p<0.05$ (t-tests).

\section{Discussion}

The MYB transcription factors have been reported to be one of the largest gene families in plants and play important roles in plant development, metabolism, and various stress responses [3]. In the current study, BLASTP searches were performed to identify the MYB members in potato. The identified potato $M Y B$ genes were studied via analyses of phylogeny, gene structure and motif organization, syntenic analysis, chromosomal distribution, duplication event, and expression profiles. Moreover, the homologous counterparts between Arabidopsis and potato R2R3-MYB genes were investigated to predict their potential functions. 
A total of 233 MYB genes were identified in the potato genome, including 119 R1-MYB, 112 R2R3-MYB, and 2 R1R2R3 MYB members. Segmental and tandem duplication events were found to play important roles in the expansion of the MYB family in potato. A total of 31 segmental duplication pairs and 10 tandem duplication pairs were identified, with all of these duplicated pairs sharing the $\mathrm{Ka} / \mathrm{Ks}$ ratios $<1$, suggesting that these duplicated StMYB genes might have undergone purifying selection and might have maintained conserved functions during evolution. In some occasions, different cis-elements were detected on promoter regions of the duplicated gene pairs such as $S t M Y B 030 / S t M Y B 077$, implying the diversity of potential functions.

As the major MYB subgroup in plant, R2R3-MYB members have evolved from the R1R2R3-MYB ancestors by losing their R1 repeat [5]. R2R3-MYB members are featured by two MYB repeats which function in the DNA binding. The sequence features of the R2 and R3 repeat were highly conserved between potato and Arabidopsis. The similar gene structure and motif organization were observed in R2R3-MYB members within the same subgroup, supporting the results of phylogenetic analysis. The subgroup 29 only harbored R2R3-MYB members from potato, implying the member of this subgroup may have arisen after the divergence of potato and Arabidopsis. Interestingly, except StMYB219 and StMYB233, all genes from subgroup 29 were found in five clusters, indicating tandem duplication events may have played a significant role in the expansion of this subgroup during evolution.

A number of R2R3-MYB members have been characterized to participate in regulating plant development. In subgroup18, AtMYB33 and AtMYB65 have been reported to facilitate anther and pollen development [10]. StMYB001, StMYB127, StMYB137, and StMYB218 were clustered together with AtMYB33/AtMYB65 in subgroup 18 (Figure 2) and all of these four MYB encoding genes showed abundant expression in flowers (Figure 7), suggesting possible involvement of these homologous members in flower development. Similarly, AtMYB68 in subgroup 14 was reported to specifically regulate root growth [12], and its potato homologs StMYB076 and StMYB132 were found to be highly expressed in the root (Figures 7 and 9A). In subgroup 15, AtMYB0 and AtMYB23 were reported to function in the origination of trichome in shoots [13,14], whereas their potato homolog StMYB133 (Figures 7 and 9A) was found to be expressed in stem, stolon, and tuber. All these data suggested functional conservation between homologous R2R3-MYB members from Arabidopsis and potato.

In Arabidopsis, AtMYB11/PFG2, AtMYB12/PFG1, and AtMYB111/PFG3 in subgroup 7 have been reported to control flavonol biosynthesis in all tissues [17], and StMYB005, StMYB104, and StMYB108 were close homologs of these PFG members (Figure 2). In addition, StMYB005 and StMYB108 were predicted to form syntenic gene pairs with AtMYB11 (Figure 3 and Supplementary Table S3). StMYB005/StMYB108 genes were identified as a segmental duplication pair (Figure 5). However, StMYB005, StMYB104, and StMYB108 exhibited different expression patterns. StMYB005 was found to be expressed in stem and leaf, whereas $S t M Y B 108$ was expressed in flower and leaf (Figure 7), indicating possible functional divergence between these R2R3-MYB homologs.

$A t M Y B 2$ has been reported to be up-regulated in senescent leaves and involved in regulation of leaf senescence [16]. AtMYB108 was found to bind to specific regions of the ANAC003 promoter to form a MYB-NAC regulatory network, which participated in leaf senescence regulation [56]. In this study, the phylogenetic analysis revealed StMYB101, StMYB003, StMYB227, StMYB228, StMYB229, StMYB057, and StMYB083 were clustered together with AtMYB2 and AtMYB108 in subgroup 20 (Figure 2). The qRT-PCR results indicated that these potato MYB genes, except for StMYB228, were highly expressed in senescent leaves (Figure 9), suggesting that these homologous genes might also participate in the regulation of leaf senescence. Interestingly, although StMYB227 and StMYB228 were predicted to have arisen from tandem duplication events (Figure 4), StMYB227 was highly expressed in senescent leaves but StMYB228 was not (Figure 9), indicating functional divergence between these duplicated genes in regulating leaf senescence. Notably, StMYB003 and StMYB229 were found to be induced by drought stress and StMYB101 was responsive to heat (Figure 8), suggesting that these genes may function in coordinating leaf senescence and stress responses in potato. 
A large number of R2R3-MYB family members were found to confer tolerance to abiotic and biotic stresses in plants [2]. In subgroup1, AtMYB30, AtMYB60, and AtMYB96, which have been reported to be involved in abiotic stress responses [24,25,27], were clustered together with StMYB021, StMYB034, StMYB055, StMYB123, and StMYB189 (Figure 2). In agreement with their Arabidopsis homologs, the potato genes StMYB021, StMYB055, and StMYB123 were found to be induced by salt treatments and StMYB189 was induced by heat/wounding (Figures 8 and 9B). StMYB021/StMYB034 was a segmental duplication pair but unlike StMYB021, StMYB034 was not responsive to any of the testes stress treatments (Figure 5). Functional divergence might have occurred within this pair. In subgroup 2, AtMYB15 was reported to mediate environmental signals and stresses tolerance $[30,31]$ and potato genes StMYB037, StMYB105, StMYB118, and StMYB168 in the same subgroup were induced by at least one stress treatment, implying that the potato R2R3-MYB members in this subgroup may also confer stress tolerance (Figure 8).

Also, in subgroup22, StMYB071, StMYB072, StMYB030, and StMYB077 were clustered together with Arabidopsis members AtMYB44, AtMYB70, AtMYB73, and AtMYB77 (Figure 2), which have been reported to function in regulating ABA-mediated stomatal closure and abiotic stress responses [29]. In addition, StMYB030/StMYB077 formed syntenic gene pairs with AtMYB44 (Figure 3 and Supplementary Table S3). Both StMYB030 and StMYB077 possessed ABRE elements in their promoters (Figure 6), suggesting that they might be involved in ABA signaling and stress response. Furthermore, StMYB030 was significantly induced by salt and drought treatments (Figures 8 and 9B) and the StMYB030-GFP fusion protein was found to be localized in the nucleus, suggesting that StMYB030 may act as a transcription factor regulating stress responses (Figure 10). Indeed, the overexpression analysis indicated that StMYB030 could confer salt tolerance in transgenic Arabidopsis (Figure 11).

\section{Conclusions}

In summary, a systematic investigation was performed to identify and characterize the MYB family members in potato. The characterization of the potato MYB family members provided insights into the evolutionary relationship between MYB family members. Notably, the homologous counterparts between Arabidopsis and potato MYB members were found to likely play conserved roles in regulating plant development and stress responses. Notably, the StMYB030 gene was found to be induced by salt and drought stress treatments and able to confer salt tolerance in transgenic Arabidopsis. The results provided here could be helpful in the future exploration of the biological functions of these potato MYB genes.

Supplementary Materials: The following are available online at http://www.mdpi.com/2218-273X/9/8/317/s1, Supplementary Table S1. The qRT-PCR primers used in this study; Supplementary Table S2. The detail information of identified potato MYB family members; Supplementary Table S3. The syntenic pairs between potato and other five plant species; Supplementary Table S4. The detail information of segmental and tandem duplication gene pairs; Supplementary Table S5. The detail information of the induced StMYB gene under various stress treatments; Supplementary Figure S1. The motif and gene structure organizations of StMYB members; Supplementary Figure S2. The putative conserved motifs in StMYB proteins; Supplementary Figure S3. The expression level of StMYB030 gene in wildtype and two overexpression lines, the ratios of gene expression level were calculated relative to the wildtype.

Author Contributions: X.L. and C.G. conducted the research and drafted the manuscript. S.A., Q.W., J.Y. and C.L. assisted in data collection and analysis. Y.G. conceived the research and drafted the manuscript. All authors read and approved this final manuscript.

Funding: This research was financially supported by the National Natural Science Foundation of China (31571494), the Agricultural Science and Technology Innovation Program (ASTIP-TRIC02) and the Fundamental Research Funds for Central Non-profit Scientific Institution (Y2017JC27).

Conflicts of Interest: The authors declare no conflict of interest.

\section{References}

1. Qu, L.J.; Zhu, Y.X. Transcription factor families in Arabidopsis: Major progress and outstanding issues for future research. Curr. Opin. Plant Biol. 2006, 9, 544-549. [CrossRef] [PubMed] 
2. Martin, C.; Paz-Ares, J. MYB transcription factors in plants. Trends Genet. 1997, 13, 67-73. [CrossRef]

3. Dubos, C.; Stracke, R.; Grotewold, E.; Weisshaar, B.; Martin, C.; Lepiniec, L. MYB transcription factors in Arabidopsis. Trends Plant Sci. 2010, 15, 573-581. [CrossRef] [PubMed]

4. Ogata, K.; Kanei-Ishii, C.; Sasaki, M.; Hatanaka, H.; Nagadoi, A.; Enari, M.; Nakamura, H.; Nishimura, Y.; Ishii, S.; Sarai, A. The cavity in the hydrophobic core of MYB DNA-binding domain is reserved for DNA recognition and trans-activation. Nat. Struct. Mol. Biol. 1996, 3, 178-187. [CrossRef]

5. Jiang, C.; Gu, J.; Chopra, S.; Gu, X.; Peterson, T. Ordered origin of the typical two-and three-repeat Myb genes. Gene 2004, 326, 13-22. [CrossRef] [PubMed]

6. Roy, S. Function of MYB domain transcription factors in abiotic stress and epigenetic control of stress response in plant genome. Plant Signal. Behav. 2016, 11, e1117723. [CrossRef] [PubMed]

7. Allan, A.C.; Hellens, R.P.; Laing, W.A. MYB transcription factors that colour our fruit. Trends Plant Sci. 2008, 13, 99-102. [CrossRef] [PubMed]

8. Song, S.; Qi, T.; Huang, H.; Ren, Q.; Wu, D.; Chang, C.; Peng, W.; Liu, Y.; Peng, J.; Xie, D. The jasmonate-ZIM domain proteins interact with the R2R3-MYB transcription factors MYB21 and MYB24 to affect jasmonate-regulated stamen development in Arabidopsis. Plant Cell 2011. [CrossRef]

9. Cheng, H.; Song, S.; Xiao, L.; Soo, H.M.; Cheng, Z.; Xie, D.; Peng, J. Gibberellin acts through jasmonate to control the expression of MYB21, MYB24, and MYB57 to promote stamen filament growth in Arabidopsis. PLoS Genet. 2009, 5, e1000440. [CrossRef]

10. Millar, A.A.; Gubler, F. The Arabidopsis GAMYB-like genes, MYB33 and MYB65, are MicroRNA-regulated genes that redundantly facilitate anther development. Plant Cell 2005, 17, 705-721. [CrossRef]

11. Huang, H.; Gao, H.; Liu, B.; Qi, T.; Tong, J.; Xiao, L.; Xie, D.; Song, S. Arabidopsis MYB24 Regulates Jasmonate-Mediated Stamen Development. Front. Plant Sci. 2017, 8, 1525. [CrossRef] [PubMed]

12. Feng, C.; Andreasson, E.; Maslak, A.; Mock, H.P.; Mattsson, O.; Mundy, J. Arabidopsis MYB68 in development and responses to environmental cues. Plant Sci. 2004, 167, 1099-1107. [CrossRef]

13. Kirik, V.; Schnittger, A.; Radchuk, V.; Adler, K.; Hülskamp, M.; Bäumlein, H. Ectopic expression of the Arabidopsis AtMYB23 gene induces differentiation of trichome cells. Dev. Biol. 2001, 235, 366-377. [CrossRef] [PubMed]

14. Kang, Y.H.; Kirik, V.; Hulskamp, M.; Nam, K.H.; Hagely, K.; Lee, M.M.; Schiefelbein, J. The MYB23 gene provides a positive feedback loop for cell fate specification in the Arabidopsis root epidermis. Plant Cell 2009, 21, 1080-1094. [CrossRef] [PubMed]

15. Guo, Y. Towards systems biological understanding of leaf senescence. Plant Mol. Biol. 2013, 82, 519-528. [CrossRef] [PubMed]

16. Guo, Y.; Gan, S.S. AtMYB2 regulates whole plant senescence by inhibiting cytokinin-mediated branching at late stages of development in Arabidopsis. Plant Physiol. 2011, 156, 1612-1619. [CrossRef]

17. Stracke, R.; Ishihara, H.; Huep, G.; Barsch, A.; Mehrtens, F.; Niehaus, K.; Weisshaar, B. Differential regulation of closely related R2R3-MYB transcription factors controls flavonol accumulation in different parts of the Arabidopsis thaliana seedling. Plant J. 2007, 50, 660-677. [CrossRef] [PubMed]

18. Teng, S.; Keurentjes, J.; Bentsink, L.; Koornneef, M.; Smeekens, S. Sucrose-specific induction of anthocyanin biosynthesis in Arabidopsis requires the MYB75/PAP1 gene. Plant Physiol. 2005, 139, 1840-1852. [CrossRef]

19. Gonzalez, A.; Zhao, M.; Leavitt, J.M.; Lloyd, A.M. Regulation of the anthocyanin biosynthetic pathway by the TTG1/bHLH/Myb transcriptional complex in Arabidopsis seedlings. Plant J. 2008, 53, 814-827. [CrossRef]

20. Zhong, R.; Lee, C.; Zhou, J.; McCarthy, R.L.; Ye, Z.H. A battery of transcription factors involved in the regulation of secondary cell wall biosynthesis in Arabidopsis. Plant Cell 2008, 20, 2763-2782. [CrossRef]

21. Shi, D.; Ren, A.; Tang, X.; Qi, G.; Xu, Z.; Chai, G.; Hu, R.; Zhou, G.; Kong, Y. MYB52 Negatively Regulates Pectin Demethylesterification in Seed Coat Mucilage. Plant Physiol. 2018, 176, 2737-2749. [CrossRef] [PubMed]

22. Frerigmann, H.; Glawischnig, E.; Gigolashvili, T. The role of MYB34, MYB51 and MYB122 in the regulation of camalexin biosynthesis in Arabidopsis thaliana. Front. Plant Sci. 2015, 6, 654. [CrossRef] [PubMed]

23. Frerigmann, H.; Gigolashvili, T. MYB34, MYB51, and MYB122 distinctly regulate indolic glucosinolate biosynthesis in Arabidopsis thaliana. Mol. Plant 2014, 7, 814-828. [CrossRef] [PubMed]

24. Oh, J.E.; Kwon, Y.; Kim, J.H.; Noh, H.; Hong, S.W.; Lee, H. A dual role for MYB60 in stomatal regulation and root growth of Arabidopsis thaliana under drought stress. Plant Mol. Biol. 2011, 77, 91-103. [CrossRef] [PubMed] 
25. Seo, P.J.; Xiang, F.; Qiao, M.; Park, J.Y.; Lee, Y.N.; Kim, S.G.; Lee, Y.H.; Park, W.J.; Park, C.-M. The MYB96 transcription factor mediates abscisic acid signaling during drought stress response in Arabidopsis. Plant Physiol. 2009, 151, 275-289. [CrossRef] [PubMed]

26. Seo, P.J.; Park, C.M. MYB96-mediated abscisic acid signals induce pathogen resistance response by promoting salicylic acid biosynthesis in Arabidopsis. New Phytol. 2010, 186, 471-483. [CrossRef] [PubMed]

27. Marino, D.; Froidure, S.; Canonne, J.; Khaled, S.B.; Khafif, M.; Pouzet, C.; Jauneau, A.; Roby, D.; Rivas, S. Arabidopsis ubiquitin ligase MIEL1 mediates degradation of the transcription factor MYB30 weakening plant defence. Nat. Commun. 2013, 4, 1476. [CrossRef] [PubMed]

28. Reyes, J.L.; Chua, N.H. ABA induction of miR159 controls transcript levels of two MYB factors during Arabidopsis seed germination. Plant J. 2007, 49, 592-606. [CrossRef]

29. Jung, C.; Seo, J.S.; Han, S.W.; Koo, Y.J.; Kim, C.H.; Song, S.I.; Nahm, B.H.; Do Choi, Y.; Cheong, J.J. Overexpression of AtMYB44 enhances stomatal closure to confer abiotic stress tolerance in transgenic Arabidopsis. Plant Physiol. 2008, 146, 623-635. [CrossRef] [PubMed]

30. Ding, Z.; Li, S.; An, X.; Liu, X.; Qin, H.; Wang, D. Transgenic expression of MYB15 confers enhanced sensitivity to abscisic acid and improved drought tolerance in Arabidopsis thaliana. J. Genet. Genom. 2009, 36, 17-29. [CrossRef]

31. Agarwal, M.; Hao, Y.; Kapoor, A.; Dong, C.-H.; Fujii, H.; Zheng, X.; Zhu, J.K. A R2R3 type MYB transcription factor is involved in the cold regulation of CBF genes and in acquired freezing tolerance. J. Biol. Chem. 2006, 281, 37636-37645. [CrossRef] [PubMed]

32. Abe, H.; Urao, T.; Ito, T.; Seki, M.; Shinozaki, K.; Yamaguchi-Shinozaki, K. Arabidopsis AtMYC2 (bHLH) and AtMYB2 (MYB) function as transcriptional activators in abscisic acid signaling. Plant Cell 2003, 15, 63-78. [CrossRef] [PubMed]

33. Devaiah, B.N.; Madhuvanthi, R.; Karthikeyan, A.S.; Raghothama, K.G. Phosphate starvation responses and gibberellic acid biosynthesis are regulated by the MYB62 transcription factor in Arabidopsis. Mol. Plant 2009, 2, 43-58. [CrossRef] [PubMed]

34. Mengiste, T.; Chen, X.; Salmeron, J.; Dietrich, R. The BOTRYTIS SUSCEPTIBLE1 gene encodes an R2R3MYB transcription factor protein that is required for biotic and abiotic stress responses in Arabidopsis. Plant Cell 2003, 15, 2551-2565. [CrossRef] [PubMed]

35. Luo, H.; Laluk, K.; Lai, Z.; Veronese, P.; Song, F.; Mengiste, T. The Arabidopsis Botrytis Susceptible1 Interactor defines a subclass of RING E3 ligases that regulate pathogen and stress responses. Plant Physiol. 2010, 154, 1766-1782. [CrossRef]

36. De Vos, M.; Denekamp, M.; Dicke, M.; Vuylsteke, M.; Van Loon, L.; Smeekens, S.C.; Pieterse, C.M. The Arabidopsis thaliana Transcription Factor AtMYB102 Functions in Defense against the Insect Herbivore Pieris rapae. Plant Signal. Behav. 2006, 1, 305-311. [CrossRef]

37. Segarra, G.; Van der Ent, S.; Trillas, I.; Pieterse, C. MYB72, a node of convergence in induced systemic resistance triggered by a fungal and a bacterial beneficial microbe. Plant Biol. 2009, 11, 90-96. [CrossRef]

38. Zhu, L.; Guo, J.; Ma, Z.; Wang, J.; Zhou, C. Arabidopsis Transcription Factor MYB102 Increases Plant Susceptibility to Aphids by Substantial Activation of Ethylene Biosynthesis. Biomolecules 2018, 8, 39. [CrossRef]

39. Katiyar, A.; Smita, S.; Lenka, S.K.; Rajwanshi, R.; Chinnusamy, V.; Bansal, K.C. Genome-wide classification and expression analysis of MYB transcription factor families in rice and Arabidopsis. BMC Genom. 2012, 13, 544. [CrossRef]

40. Li, Z.; Peng, R.; Tian, Y.; Han, H.; Xu, J.; Yao, Q. Genome-wide Identification and analysis of the MYB transcription factor superfamily in Solanum lycopersicum. Plant Cell Physiol. 2016, 57, 1657-1677. [CrossRef]

41. Matus, J.T.; Aquea, F.; Arce-Johnson, P. Analysis of the grape MYB R2R3 subfamily reveals expanded wine quality-related clades and conserved gene structure organization across Vitis and Arabidopsis genomes. BMC Plant Biol. 2008, 8, 83. [CrossRef] [PubMed]

42. Du, H.; Feng, B.-R.; Yang, S.S.; Huang, Y.-B.; Tang, Y.-X. The R2R3-MYB transcription factor gene family in maize. PLoS ONE 2012, 7, e37463. [CrossRef] [PubMed]

43. Cao, Y.; Han, Y.; Li, D.; Lin, Y.; Cai, Y. MYB transcription factors in chinese pear (Pyrus bretschneideri Rehd.): Genome-wide identification, classification, and expression profiling during fruit development. Front. Plant Sci. 2016, 7, 577. [CrossRef] [PubMed] 
44. Zhang, T.; Zhao, Y.; Wang, Y.; Liu, Z.; Gao, C. Comprehensive analysis of MYB gene family and their expressions under abiotic stresses and hormone treatments in Tamarix hispida. Front. Plant Sci. 2018, 9. [CrossRef] [PubMed]

45. Mmadi, M.; Dossa, K.; Wang, L.; Zhou, R.; Wang, Y.; Cisse, N.; Sy, M.; Zhang, X. Functional characterization of the versatile $M Y B$ gene family uncovered their important roles in plant development and responses to drought and waterlogging in sesame. Genes 2017, 8, 362. [CrossRef] [PubMed]

46. Bailey, T.L.; Boden, M.; Buske, F.A.; Frith, M.; Grant, C.E.; Clementi, L.; Ren, J.; Li, W.W.; Noble, W.S. MEME SUITE: Tools for motif discovery and searching. Nucleic Acids Res. 2009, 37, W202-W208. [CrossRef] [PubMed]

47. Hu, B.; Jin, J.; Guo, A.Y.; Zhang, H.; Luo, J.; Gao, G. GSDS 2.0: An upgraded gene feature visualization server. Bioinformatics 2014, 31, 1296-1297. [CrossRef] [PubMed]

48. Li, X.; Ahmad, S.; Guo, C.; Yu, J.; Cao, S.; Gao, X.; Li, W.; Li, H.; Guo, Y. Identification and Characterization of LRR-RLK Family Genes in Potato Reveal Their Involvement in Peptide Signaling of Cell Fate Decisions and Biotic/Abiotic Stress Responses. Cells 2018, 7, 120. [CrossRef]

49. Xie, T.; Chen, C.; Li, C.; Liu, J.; Liu, C.; He, Y. Genome-wide investigation of WRKY gene family in pineapple: Evolution and expression profiles during development and stress. BMC Genom. 2018, 19, 490. [CrossRef]

50. Librado, P.; Rozas, J. DnaSP v5: A software for comprehensive analysis of DNA polymorphism data. Bioinformatics 2009, 25, 1451-1452. [CrossRef]

51. Consortium, P.G.S. Genome sequence and analysis of the tuber crop potato. Nature 2011, 475, 189. [CrossRef]

52. Singh, A.K.; Sharma, V.; Pal, A.K.; Acharya, V.; Ahuja, P.S. Genome-wide organization and expression profiling of the NAC transcription factor family in potato (Solanum tuberosum L.). DNA Res. 2013, 20, 403-423. [CrossRef] [PubMed]

53. Li, X.; Ahmad, S.; Ali, A.; Guo, C.; Li, H.; Yu, J.; Zhang, Y.; Gao, X.; Guo, Y. Characterization of Somatic Embryogenesis Receptor-Like Kinase 4 as a Negative Regulator of Leaf Senescence in Arabidopsis. Cells 2019, 8, 50. [CrossRef] [PubMed]

54. Li, X.; Hamyat, M.; Liu, C.; Ahmad, S.; Gao, X.; Guo, C.; Wang, Y.; Guo, Y. Identification and characterization of the WOX family genes in five Solanaceae species reveal their conserved roles in peptide signaling. Genes 2018, 9, 260. [CrossRef] [PubMed]

55. Zhang, X.; Henriques, R.; Lin, S.S.; Niu, Q.W.; Chua, N.H. Agrobacterium-mediated transformation of Arabidopsis thaliana using the floral dip method. Nat. Protoc. 2006, 1, 641. [CrossRef] [PubMed]

56. Chou, M.L.; Liao, W.Y.; Wei, W.C.; Li, A.Y.S.; Chu, C.Y.; Wu, C.L.; Liu, C.L.; Fu, T.H.; Lin, L.F. The Direct Involvement of Dark-Induced Tic55 Protein in Chlorophyll Catabolism and Its Indirect Role in the MYB108-NAC Signaling Pathway during Leaf Senescence in Arabidopsis thaliana. Int. J. Mol. Sci. 2018, 19, 1854. [CrossRef] [PubMed]

(C) 2019 by the authors. Licensee MDPI, Basel, Switzerland. This article is an open access article distributed under the terms and conditions of the Creative Commons Attribution (CC BY) license (http://creativecommons.org/licenses/by/4.0/). 